\title{
Neuroprotection in epilepsy: the role of antiepileptic drugs Vasileios Kimiskidis
}

\author{
Address: 3rd Department of Psychiatry, Aristotle University of Thessaloniki, Greece \\ from International Society on Brain and Behaviour: 3rd International Congress on Brain and Behaviour \\ Thessaloniki, Greece. 28 November - 2 December 2007 \\ Published: 17 April 2008 \\ Annals of General Psychiatry 2008, 7(Suppl I):S74 doi:I0.II86/I744-859X-7-SI-S74
}

This abstract is available from: http://www.annals-general-psychiatry.com/content/7/SI/S74

(C) 2008 Kimiskidis; licensee BioMed Central Ltd.

During the last 15 years, accumulating experimental and clinical evidence suggested that the epileptic brain may undergo significant modifications as a result of recurrent seizures or due to the underlying seizure etiology. This concept of epilepsy as an evolving neurodegenerative process is best exemplified by temporal lobe epilepsy. According to current thinking, in patients with temporal lobe epilepsy a triggering factor, such as complicated febrile seizures or head trauma, sets in motion the process of epileptogenesis, that is a cascade of events leading to a gradual increase of brain excitability and eventually the occurrence of spontaneous seizures. In a subset of patients, this process further evolves due to a complex interaction of neurodegenerative and neuroplastic changes brought about by recurrent seizures. At a clinical level, this evolving process becomes evident by the onset of pharmacoresistant epileptic seizures sometimes accompanied by cognitive decline. The current pharmacological treatment of epilepsy is directed towards suppressing epileptic seizures and therefore it is essentially a symptomatic approach. The recent unraveling of the cellular mechanisms underlying epileptogenesis, however, has led to the discovery of pharmacological agents with neuroprotective and antiepileptogenic properties, which include a number of clinically available antiepileptic drugs. These agents hold promise as disease modification strategies which may ultimately improve the long-term outcome of epileptic patients by mitigating cognitive compromise and preventing the transition to pharmacoresistance. This presentation reviews the available evidence regarding the neuroprotective properties of antiepileptic drugs and discusses some methodological difficulties in designing clinical trials with a view to investigating neuroprotective strategies in epilepsy. 